University of Wollongong

Research Online

Faculty of Engineering and Information

Faculty of Engineering and Information

Sciences - Papers: Part A

Sciences

$1-1-2013$

Experimental demonstration and analysis of compact silicon-nanowirebased couplers

Hongqiang Li

Tianjin Polytechnic University, lihongqiang@tjpu.edu.cn

Xiaye Dong

Tianjin Polytechnic University

Yaoting Bai

Tianjin Polytechnic University

Enbang Li

University of Sydney, enbang@uow.edu.au

Yang Li

Tianjin Polytechnic University

See next page for additional authors

Follow this and additional works at: https://ro.uow.edu.au/eispapers

Part of the Engineering Commons, and the Science and Technology Studies Commons

Research Online is the open access institutional repository for the University of Wollongong. For further information contact the UOW Library: research-pubs@uow.edu.au 


\title{
Experimental demonstration and analysis of compact silicon-nanowire-based couplers
}

\begin{abstract}
Compact $2 \times 2$ couplers based on silicon nanowires are fabricated and tested. They include a directional (X) coupler, a cross-gap coupler (CGC), and a multimode interference (MMI) coupler. The length of the $\mathrm{X}$ coupler's parallel film waveguide is $1 \mu \mathrm{m}$. The theoretical minimum excess loss of the $X$ coupler is 0.73 $\mathrm{dB}$, whereas its experimental value is $1.0817 \mathrm{~dB}$. CGC has a coupling region length of $24 \mu \mathrm{m}$. The minimum excess loss of CGC, which is $0.6 \mathrm{~dB}$ in theory, is experimentally determined to be $0.6737 \mathrm{~dB}$. Taper waveguides are used as input/output waveguides for the MMI coupler. The footprint of the MMI region is only $6 \times 57 \mu \mathrm{m} 2$. The excess loss of the MMl coupler is theoretically $0.46 \mathrm{~dB}$, but its experimental value is $0.5423 \mathrm{~dB}$. The experimental nonuniformity of the MMl coupler is $0.0063 \mathrm{~dB}$ when the center wavelength is $1.55 \mu \mathrm{m}$. The maximum excess loss of the MMl coupler is $0.8233 \mathrm{~dB}$ in the wavelength range of 1.52 to $1.58 \mu \mathrm{m}$. The simulated and experimental results show that a small $2 \times 2$ MMI coupler that is suitable for optoelectronic integration exhibits lower excess loss, wider bandwidth, and better uniformity.
\end{abstract}

\section{Keywords}

compact, analysis, couplers, demonstration, nanowire, experimental, silicon

\section{Disciplines}

Engineering | Science and Technology Studies

\section{Publication Details}

Li, H., Dong, X., Bai, Y., Li, E., Li, Y., Liu, Y. \& Zhou, W. (2013). Experimental demonstration and analysis of compact silicon-nanowire-based couplers. Optical Engineering, 52 (6), 064003-1-064003-6.

\section{Authors}

Hongqiang Li, Xiaye Dong, Yaoting Bai, Enbang Li, Yang Li, Yu Liu, and Wenqian Zhou 


\section{Optical Engineering}

\section{Experimental demonstration and analysis of compact silicon-nanowire- based couplers}

Hongqiang Li

Xiaye Dong

Yaoting Bai

Enbang Li

Yang Li

Yu Liu

Wenqian Zhou 


\section{Experimental demonstration and analysis of compact silicon-nanowire-based couplers}

\author{
Hongqiang Li \\ Xiaye Dong \\ Yaoting Bai \\ Tianjin Polytechnic University \\ School of Electronics and Information Engineering \\ Tianjin 300387, China \\ E-mail: lihongqiang@tjpu.edu.cn \\ Enbang Li \\ University of Sydney \\ Centre for Ultrahigh bandwidth Devices for Optical \\ Systems \\ New South Wales 2006, Australia \\ Yang Li \\ Yu Liu \\ Wenqian Zhou \\ Tianjin Polytechnic University \\ School of Electronics and Information Engineering \\ Tianjin 300387, China
}

\begin{abstract}
Compact $2 \times 2$ couplers based on silicon nanowires are fabricated and tested. They include a directional $(X)$ coupler, a cross-gap coupler (CGC), and a multimode interference (MMI) coupler. The length of the $X$ coupler's parallel film waveguide is $1 \mu \mathrm{m}$. The theoretical minimum excess loss of the $X$ coupler is $0.73 \mathrm{~dB}$, whereas its experimental value is $1.0817 \mathrm{~dB}$. CGC has a coupling region length of $24 \mu \mathrm{m}$. The minimum excess loss of CGC, which is $0.6 \mathrm{~dB}$ in theory, is experimentally determined to be $0.6737 \mathrm{~dB}$. Taper waveguides are used as input/output waveguides for the MMl coupler. The footprint of the MMI region is only $6 \times 57 \mu \mathrm{m}^{2}$. The excess loss of the MMI coupler is theoretically $0.46 \mathrm{~dB}$, but its experimental value is $0.5423 \mathrm{~dB}$. The experimental nonuniformity of the MMI coupler is $0.0063 \mathrm{~dB}$ when the center wavelength is $1.55 \mu \mathrm{m}$. The maximum excess loss of the MMI coupler is $0.8233 \mathrm{~dB}$ in the wavelength range of 1.52 to $1.58 \mu \mathrm{m}$. The simulated and experimental results show that a small $2 \times 2 \mathrm{MMl}$ coupler that is suitable for optoelectronic integration exhibits lower excess loss, wider bandwidth, and better uniformity. (๑) 2013 Society of Photo-Optical Instrumentation Engineers (SPIE) [DOI: 10.1117/1.OE.52.6.064003]
\end{abstract}

Subject terms: optical devices; silicon-nanowire; coupler; loss; optoelectronic integration.

Paper 130438 received Mar. 19, 2013; revised manuscript received May 15, 2013; accepted for publication May 30, 2013; published online Jun. 19, 2013; corrected Jun. 21, 2013.

\section{Introduction}

In the optical fiber grating demodulation system, optical couplers have irreplaceable and important functions. The development of low-cost, high-performance optical couplers with high-level integration is necessary and urgent to meet the requirements of demodulation systems. ${ }^{1}$ In 2008, Chen $^{2}$ introduced the application of a femtosecond fiber laser to fabricate broadband directional (X) couplers inside bulk glass for general power-splitting applications within the telecom spectrum with wavelengths of 1250 to $1650 \mathrm{~nm}$; the coupling region length of the said coupler was $0.1 \mathrm{~mm}$. Simultaneously, Yang $^{3}$ designed and fabricated a $2 \times 2$ tapered multimode interference (MMI) coupler using an aircladded SU-8 rectangular waveguide with a total MMI size of approximately $5.3 \times 34.2 \mu \mathrm{m}^{2}$, an excess loss of $1.3 \mathrm{~dB}$, and a nonuniformity of $\sim 0.8 \mathrm{~dB}$. In 2010 , Tanaka ${ }^{4}$ proposed and designed a CGC based on silicon-on-insulator (SOI) technology. Meanwhile, Halir ${ }^{5}$ designed a colorless X coupler with a dispersion-engineered subwavelength structure in 2012; this coupler covered a $100 \mathrm{~nm}$ bandwidth with an imbalance of less than $0.6 \mathrm{~dB}$.

The aforementioned $2 \times 2$ couplers were applied in different optical devices. These couplers have the advantage of either a wider bandwidth or a smaller footprint. Here, we have designed a silicon-nanowire-based $\mathrm{X}$ coupler, CGC, and $2 \times 2$ MMI coupler for the array waveguide grating (AWG) demodulation integration microsystem. ${ }^{6,7}$ These couplers are used in the $\mathrm{C}$ band. Their splitting ratio should be 50:50 to obtain the maximum power for AWG. The couplers are simulated using the beam propagation method (BPM),

0091-3286/2013/\$25.00 @ 2013 SPIE with SOI as the fabrication material. The designed couplers are fabricated and tested in this study. The simulated and experimental results are compared, and their differences are analyzed. The results show that the designed couplers exhibit low loss. Specifically, the MMI coupler has the combined advantages of low loss and low nonuniformity as well as a small footprint and a wide bandwidth.

\section{Fabrication and Testing of $2 \times 2$ Couplers}

\subsection{Fabrication Process and Testing System}

SOI is selected as the material for the simulation and fabrication of the couplers. SOI is a prominent platform for microelectronics and optoelectronics, which can be used in optical device interconnections and applied in military devices. ${ }^{8,9}$ This material is superior in terms of its compatibility with silicon processing, convenient electronic or photonic integration, waveguide characteristics, facile operation in optical circuits, and radioprotection. Bent waveguides with smaller radii of curvature can be realized on SOI substrates. Extremely small devices can be fabricated on SOI substrates because of the ultrahigh contrast between the refractive indices of $\mathrm{Si}$ and $\mathrm{SiO}_{2}$.

The original equipment manufacturer of our designed coupler is Institute of Microelectronics (IME) from Singapore. The bare SOI wafer used during fabrication had the following parameters: (1) buried $\mathrm{SiO}_{2}$ with a thickness of $2 \mu \mathrm{m}$ and a refractive index of 1.45; and (2) top Si layer, with a thickness of $220 \mathrm{~nm}$ and a refractive index of 3.46. The standard fabrication process used by IME for optical couplers is schematically shown in Fig. 1. Here, 248-nm lithography is used. 
(a)
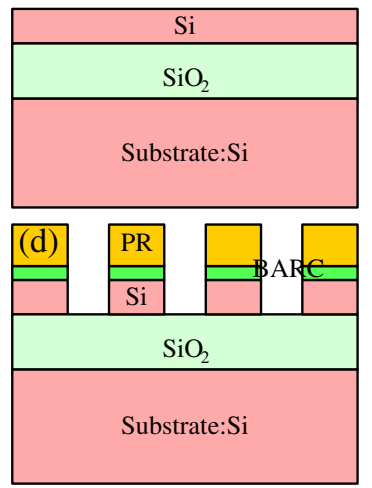
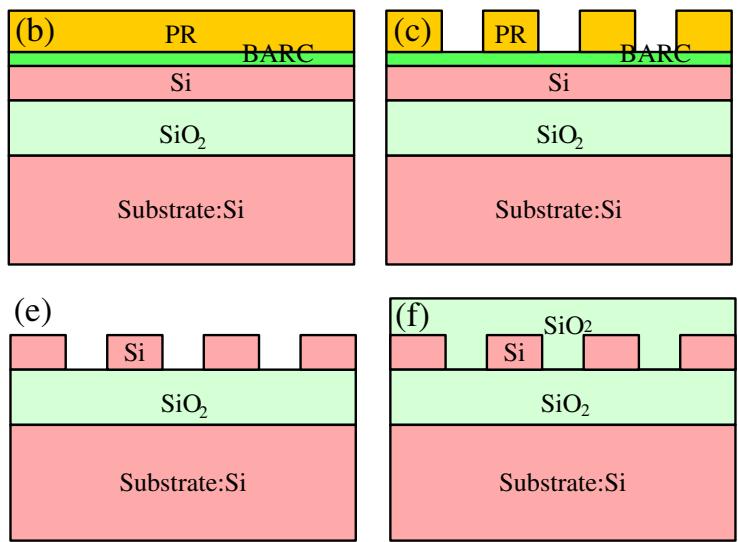

Fig. 1 Fabrication process flow used by IME for optical couplers. (a) Bare Si-substrate wafer; (b) bottom antireflective coating (BARC), photoresist (PR) coating and soft bake; (c) exposure, postexposure bake, and development; (d) BARC and Si etching; (e) PR stripping and clean-up; and (f) Si surface treatment.

A tunable laser is used on the C-band during testing. The SMF-28 with a tapered tip at one end is selected to couple the light source and the chips. The coupling efficiency of the tapered fiber and the waveguides is approximately $50 \%$. During the testing process, an infrared microscope with a camera is used to observe the output of the couplers.

\subsection{Coupler Testing}

The $\mathrm{X}$ coupler is based on the power exchange principle between two waveguides approaching each other. The coupling region of the $\mathrm{X}$ coupler periodically exchanges energy by coupling between the two parallel film waveguides. The splitting ratio can be controlled by adjusting the parameters of the coupling region. The coupling length can be expressed as:

$L=\frac{\pi}{\left(n_{e}-n_{o}\right) k_{0}}$,

where $n_{e}$ and $n_{o}$ are the effective refraction indexes of the even and odd symmetrical modes, respectively. $k_{0}$ is the vacuum vector. The coupling length for a $3 \mathrm{~dB}$ X coupler is expressed as:
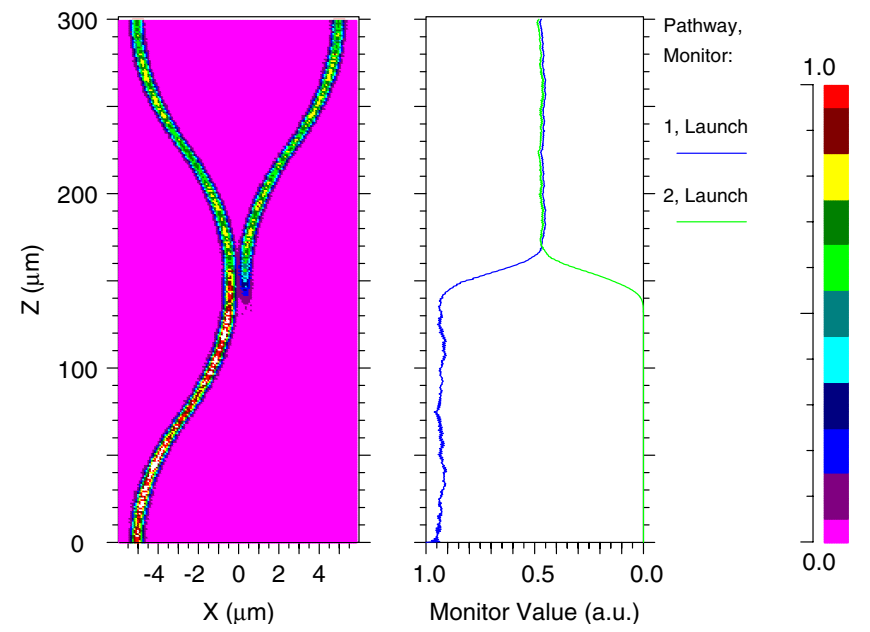

Fig. 2 Optical field and output power of the $\mathrm{X}$ coupler when $w=0.55$ and $s=0.2 \mu \mathrm{m}$. "1, Launch" represents the light power of the X coupler's input waveguide, "2, Launch" represents the light power of the $X$ coupler's coupling waveguide.
$L_{3 \mathrm{~dB}}=\frac{L}{2}=\frac{\pi}{2\left(n_{e}-n_{o}\right) k_{0}}$.

The $\mathrm{X}$ coupler is simulated via BeamPROP simulation engine, which is a part of the RSoft Photonics Suite, and is based on advanced finite-difference beam propagation techniques. The waveguide width is expressed as $w$. The distance between two parallel film waveguides is expressed as $s$. X couplers with different $w$ and $s$ are simulated, and the best simulation result is presented in Fig. 2.

The micrographs of the fabricated $X$ coupler are shown in Fig. 3. A representative charge-coupled device (CCD) image of the output field is shown in Fig. 4. When the center wavelength is $1.55 \mu \mathrm{m}$, the excess loss of the device is approximately $0.73 \mathrm{~dB}$ based on the simulation results. However, its experiment value is $1.0817 \mathrm{~dB}$. The simulated and experimental results are compared in Table 1 . CR represents the splitting ratio:

$\mathrm{CR}=\frac{P_{\mathrm{OUT} i}}{\Sigma P_{\mathrm{OUT} i}} \times 100 \%$,

where $P_{\mathrm{OUT} i}$ is the output power from the coupler's output port $i$.

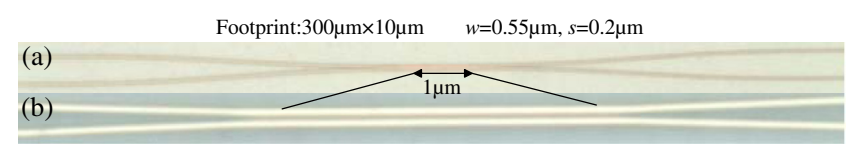

Fig. 3 Micrographs of the $X$ coupler.

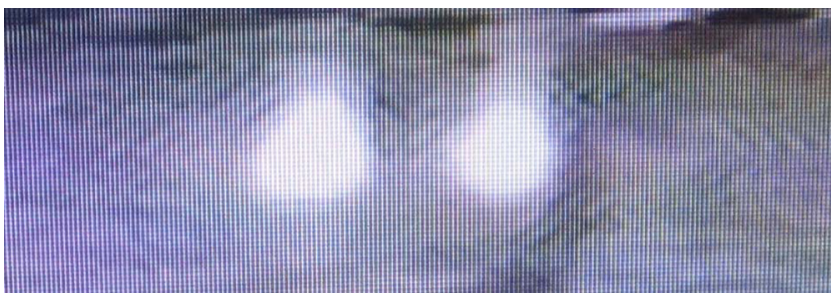

Fig. 4 Charge-coupled device (CCD) image for the output field of the $\mathrm{X}$ coupler when $w=0.55$ and $s=0.2 \mu \mathrm{m}$. 
Table 1 Comparison of $X$ coupler properties.

\begin{tabular}{lccccc}
\hline \hline & & & & & \\
& CR & UL $(d B)$ & IL1 $(d B)$ & IL2 $(d B)$ & EL $(d B)$ \\
\hline Simulation results & 0.9539 & 0.2050 & 3.8439 & 3.6389 & 0.73 \\
Experimental results & 2.0347 & 3.0850 & 2.8179 & 5.9029 & 1.0817 \\
\hline \hline
\end{tabular}

UL represents the nonuniformity:

$\mathrm{UL}=10 \lg \frac{P_{\mathrm{OUT} i}}{\Sigma P_{\mathrm{OUT} i}}$.

IL1 and IL2 represent the insert loss of the bar and cross paths, respectively:

$\mathrm{IL} i=-10 \lg \frac{P_{\mathrm{OUT} i}}{P_{\mathrm{IN}}}(\mathrm{dB})$,

where $P_{\mathrm{IN}}$ is the input power from the one of the coupler's output ports.

EL represents the excess loss:

$\mathrm{EL}=-10 \lg \frac{\Sigma P_{\mathrm{OUT} i}}{P_{\mathrm{IN}}}(\mathrm{dB})$

The difference between the simulated and experimental nonuniformity of the $\mathrm{X}$ coupler is relatively large (Table 1). This difference is caused by the fabrication tolerance because the $\mathrm{X}$ coupler is highly sensitive to the coupling length.

\subsection{CGC Testing}

A CGC is a coupler that has an $\mathrm{X}$-junction with an internal cross-sectional mirror. The gap in the CGC between the two waveguides functions as a half mirror. The principles of CGCs are similar to those of X couplers. However, these two types of couplers differ in their input/output waveguides, such that only those of CGCs are linear.

Similar to the $\mathrm{X}$ coupler, the waveguide width for the CGC system is expressed as $w$, whereas its gap is expressed as $s$. CGCs with different values for $w$ and $s$ are simulated, and the best simulation result is shown in Fig. 5 .

The micrographs and detailed parameters of the CGC are shown in Fig. 6. The CCD image of the output field is shown in Fig. 7. When the center wavelength is $1.55 \mu \mathrm{m}$, the excess loss of the device is $\sim 0.6 \mathrm{~dB}$ according to the simulations, whereas its experimental value is $0.6737 \mathrm{~dB}$. The simulated and experimental results are compared in Table 2 . This table shows that the differences between the simulated and experimental results are relatively small.

Bent waveguides with straight lines can induce a transverse coupling effect. Thus, after the connection of bent waveguides, the original coupling region becomes longer. The splitting ratio can be kept constant if the bent waveguides are followed by a reduced coupling region length. Moreover, bent waveguides are harder to fabricate. Thus, a CGC has better performance and a relatively higher fabrication tolerance than an $\mathrm{X}$ coupler with the same footprint.
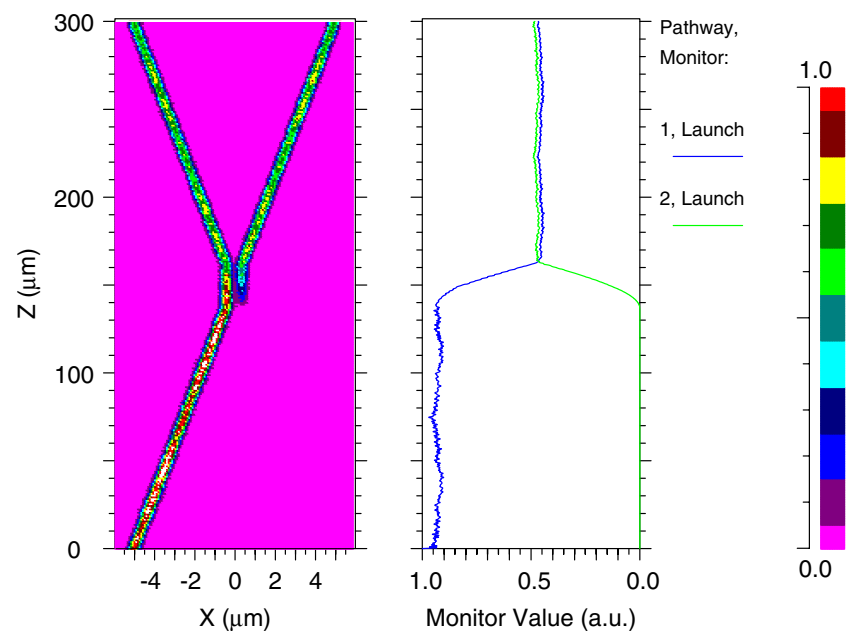

Fig. 5 Optical field and output power of cross-gap coupler (CGC) when 0.55 and $s=0.2 \mu \mathrm{m}$. "1, Launch" represents the light power of the CGC's input waveguide, "2, Launch" represents the light power of the CGC's coupling waveguide.

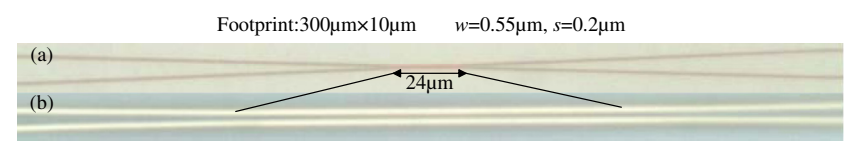

Fig. 6 Micrographs of CGC.

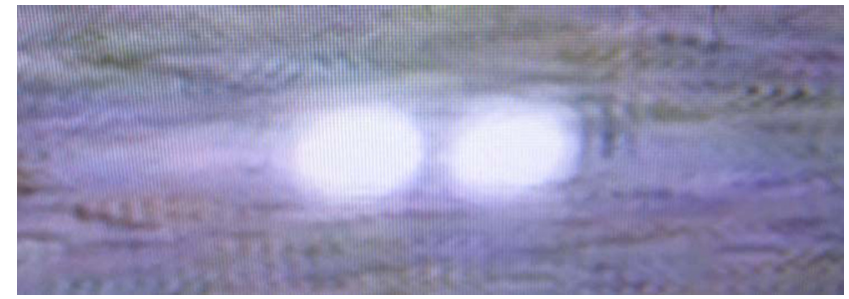

Fig. 7 CCD image for the CGC output field when $w=0.55$ and $s=0.2 \mu \mathrm{m}$.

\subsection{Testing the $2 \times 2 \mathrm{MMI}$ Couplers}

The MMI coupler is based on the self-imaging principle ${ }^{10,11}$ and has three interference mechanisms: general, paired, and symmetrical.

Paired interference is selected for this paper. Input waveguides are set in the position $\pm w_{e} / 6$ of the multimode waveguide:

$w_{e}=w+\frac{\lambda_{0}}{\pi} \cdot\left(\frac{n_{c}}{n_{r}}\right)^{2 \sigma}\left(n_{r}^{2}-n_{c}^{2}\right)^{-\frac{1}{2}}$

where $w$ is the multimode waveguide width and $\lambda_{0}$ is the center wavelength. In this equation, $\sigma=0$ for the transverse

Table 2 Comparison of cross-gap coupler (CGC) properties.

\begin{tabular}{lccccc}
\hline \hline & & & & & \\
& CR & UL $(d B)$ & IL1 $(d B)$ & IL2 (dB) & EL (dB) \\
\hline Simulation results & 0.9917 & 0.0363 & 3.6289 & 3.5924 & 0.6 \\
Experimental results & 0.9693 & 0.1355 & 3.7523 & 3.6168 & 0.6737 \\
\hline \hline
\end{tabular}


Table 3 Comparison of MMI coupler properties with different $w$.

\begin{tabular}{lccccc}
\hline \hline$w(\mu \mathrm{m})$ & $\mathrm{CR}$ & $\mathrm{UL}(\mathrm{dB})$ & $\mathrm{IL} 1(\mathrm{~dB})$ & $\mathrm{IL} 2(\mathrm{~dB})$ & $\mathrm{EL}(\mathrm{dB})$ \\
\hline 48 & 1.03 & 0.1284 & 2.9964 & 3.1256 & 0.05 \\
24 & 0.98 & 0.0877 & 3.1149 & 3.0260 & 0.06 \\
15 & 0.99 & 0.044 & 3.1220 & 3.0786 & 0.09 \\
12 & 0.985 & 0.0656 & 3.2431 & 3.1776 & 0.2 \\
6 & 1.013 & 0.06 & 3.4448 & 3.4960 & 0.46 \\
\hline \hline
\end{tabular}

electric (TE) mode, whereas $\sigma=1$ for the transverse magnetic (TM) mode. $n_{c}$ and $n_{r}$ are the effective refractive indexes of the cladding and the core, respectively.

The multimode waveguide length can be expressed as:

$L_{\mathrm{MMI}}=\frac{L_{\pi}}{2}=\frac{\pi}{2\left(\beta_{0}-\beta_{1}\right)} \approx \frac{2 n w_{e}^{2}}{3 \lambda_{0}}$,

where $L_{\pi}$ represents the coupling length of the two lowest order modes. $\beta_{0}$ and $\beta_{1}$ are the propagation constants of the lateral modes 0 and 1 , respectively.

The multimode waveguide width is expressed as $w$. A comparison of MMI coupler properties with different $w$ according to the simulation results is presented in Table 3.

MMI couplers with $w$ of 15 and $6 \mu \mathrm{m}$ are fabricated and tested in this study. The optical field and output power of the MMI coupler when $w=15 \mu \mathrm{m}$ are shown in Fig. 8 .

The micrographs of the MMI coupler when $w=15 \mu \mathrm{m}$ are shown in Fig. 9. The CCD image of the output field is shown in Fig. 10. When the center wavelength is $1.55 \mu \mathrm{m}$, the excess loss of the device is approximately $0.09 \mathrm{~dB}$ based on the simulation. However, its experimental excess loss is $0.1418 \mathrm{~dB}$. A comparison of the simulated and experimental results is presented in Table 4. From this table, the difference
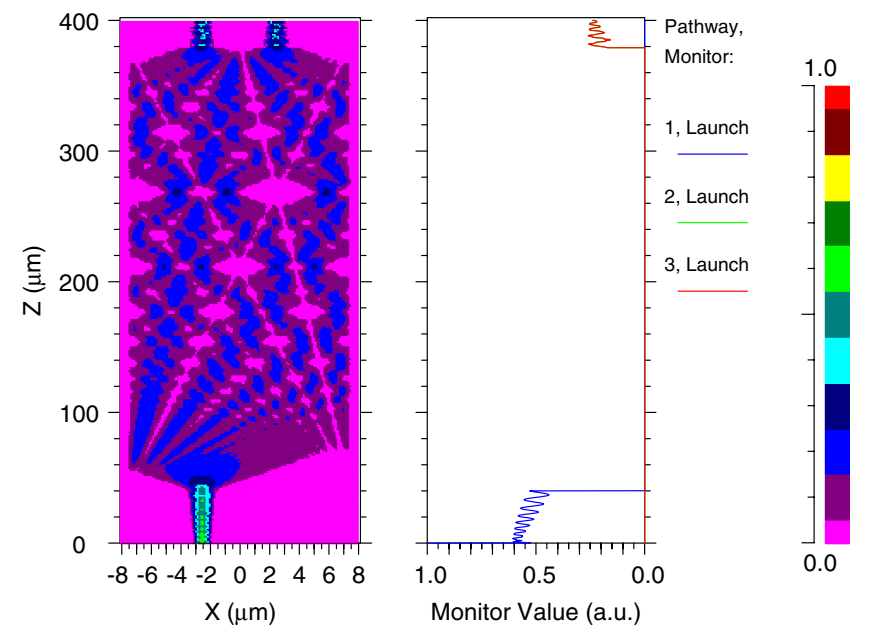

Fig. 8 Optical field and output power of the MMI coupler when $w=15 \mu \mathrm{m}$. "1, Launch" represents the light power of the MMI coupler's input waveguide, "2, Launch" represents the light power of the MMI coupler's left output waveguide, " 3 , Launch" represents the light power of the MMI coupler's right output waveguide.

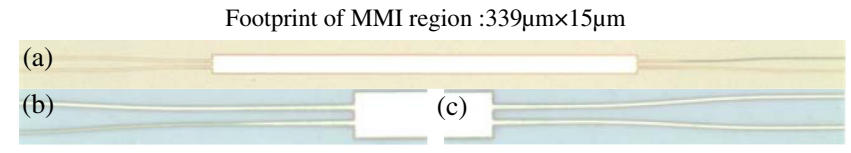

Fig. 9 Micrographs of the MMl coupler when $w=15 \mu \mathrm{m}$.

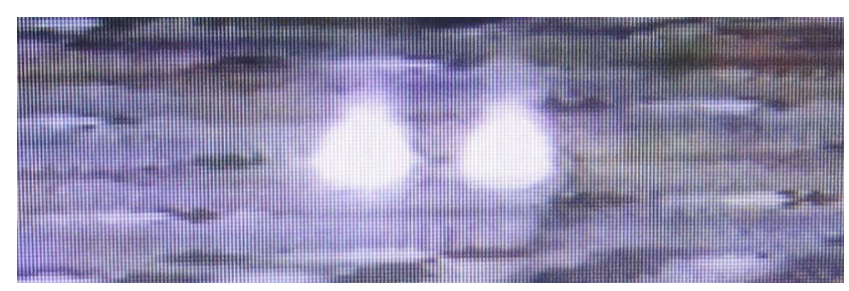

Fig. $10 \mathrm{CCD}$ image for output field of the MMI coupler when $w=15 \mu \mathrm{m}$.

Table 4 Comparison of MMl coupler properties when $w=15 \mu \mathrm{m}$.

\begin{tabular}{lclllll}
\hline \hline & $\mathrm{CR}$ & $\mathrm{UL}(\mathrm{dB})$ & $\mathrm{IL} 1(\mathrm{~dB})$ & $\mathrm{IL} 2(\mathrm{~dB})$ & $\mathrm{EL}(\mathrm{dB})$ \\
\hline Simulation results & 0.99 & 0.044 & 3.1220 & 3.0786 & 0.09 \\
Experimental results & 1.0122 & 0.0053 & 3.1495 & 3.1547 & 0.1418 \\
\hline \hline
\end{tabular}

between the simulated and experimental values is extremely small.

The output power of the MMI coupler when the center wavelength ranged from 1.52 to $1.58 \mu \mathrm{m}$ is shown in Fig. 11, where the maximum excess loss is $0.2871 \mathrm{~dB}$. P represents the output power of the light source, whereas $\mathrm{P} 0$ represents the output power of a straight waveguide. P1 and P2 represent the output power values of the coupler's bar and cross paths, respectively. The experimental results show that the designed coupler has a wide range of wavelength responses.

The optical field and output power of the MMI coupler when $w=6 \mu \mathrm{m}$ are illustrated in Fig. 12. Input/output waveguides are designed as tapered waveguides during the optimization process to make the image point clearer, to improve the splitting ratio, and to reduce loss.

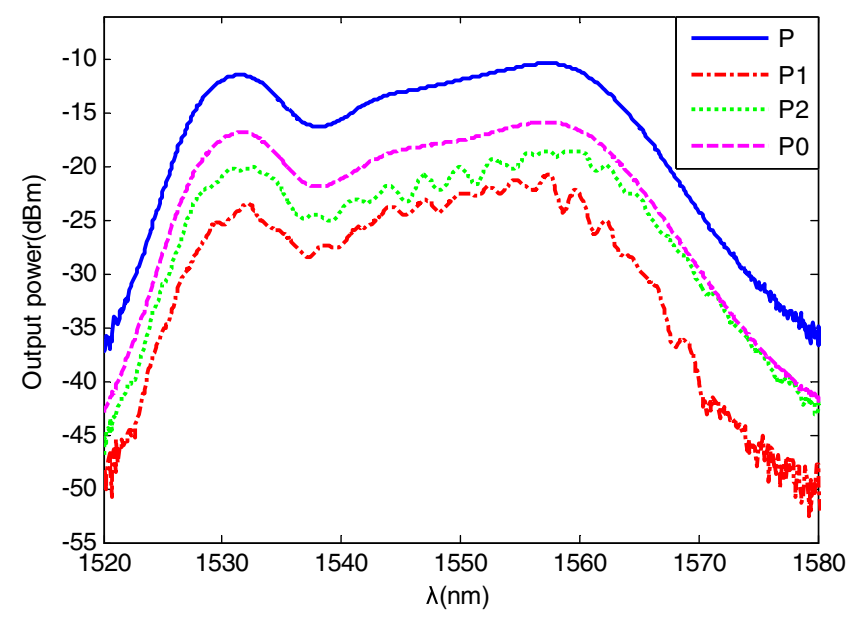

Fig. 11 Output power for MMI coupler when $w=15 \mu \mathrm{m}$. 

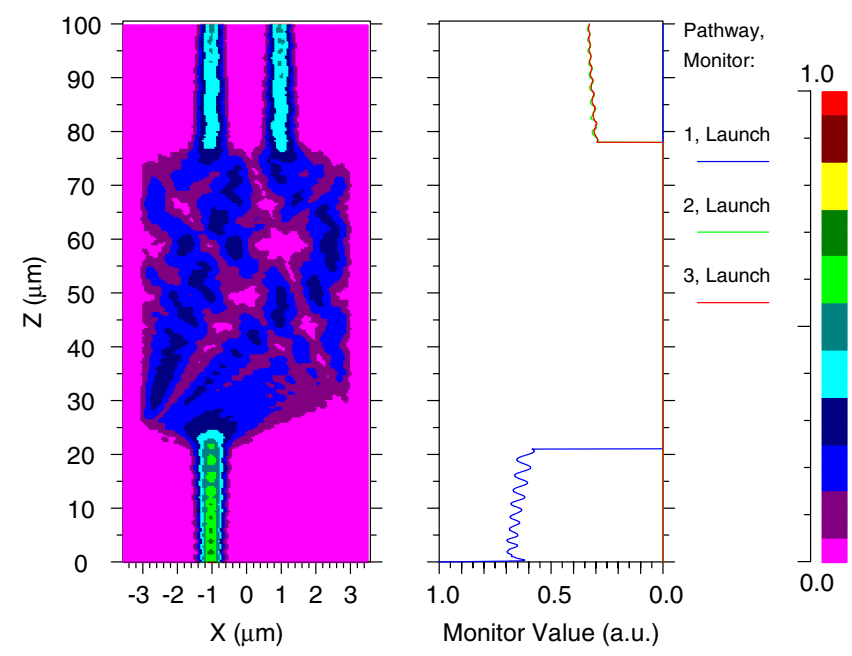

Fig. 12 Optical field and output power of the MMI coupler with a tapered input/output waveguide when $w=6 \mu \mathrm{m}$. "1, Launch" represents the light power of the MMI coupler's input waveguide, "2, Launch" represents the light power of the MMI coupler's left output waveguide, "3, Launch" represents the light power of the MMI coupler's right output waveguide.

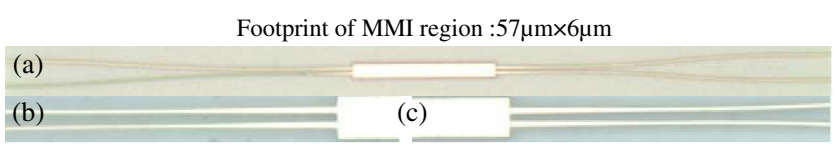

Fig. 13 Micrographs of the MMl coupler when $w=6 \mu \mathrm{m}$.

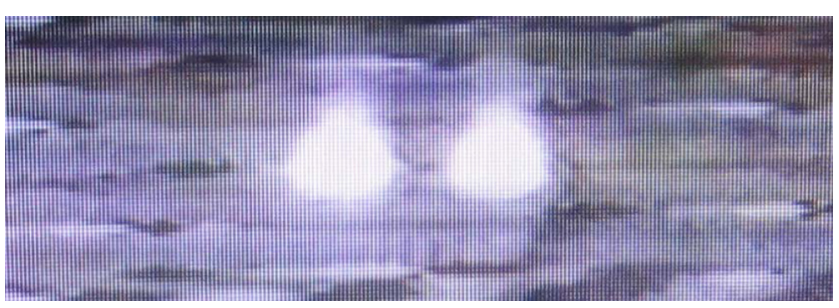

Fig. $14 \mathrm{CCD}$ image for output field of the MMI coupler when $w=6 \mu \mathrm{m}$.

The micrographs of the MMI coupler when $w=6 \mu \mathrm{m}$ are shown in Fig. 13, whereas the CCD image of its output field is presented in Fig. 14. When the center wavelength is $1.55 \mu \mathrm{m}$, the excess loss of this device is approximately $0.46 \mathrm{~dB}$ based on the simulated results, but is $0.5423 \mathrm{~dB}$ according to the actual experiment. The excess loss is larger when $w=6 \mu \mathrm{m}$ than when $w=15 \mu \mathrm{m}$ although the footprint is much smaller. The simulated and experimental results of this MMI coupler are compared in Table 5. From this table, the differences between the results of the simulation and the experiment are small.

Table 5 Comparison of MMl coupler properties when $w=6 \mu \mathrm{m}$.

\begin{tabular}{lllllll}
\hline \hline & $\mathrm{CR}$ & $\mathrm{UL}(\mathrm{dB})$ & $\mathrm{IL} 1(\mathrm{~dB})$ & $\mathrm{IL} 2(\mathrm{~dB})$ & $\mathrm{EL}(\mathrm{dB})$ \\
\hline Simulation results & 1.013 & 0.06 & 3.4448 & 3.4960 & 0.46 \\
Experimental results & 1.0122 & 0.0053 & 3.5495 & 3.5558 & 0.5423 \\
\hline \hline
\end{tabular}

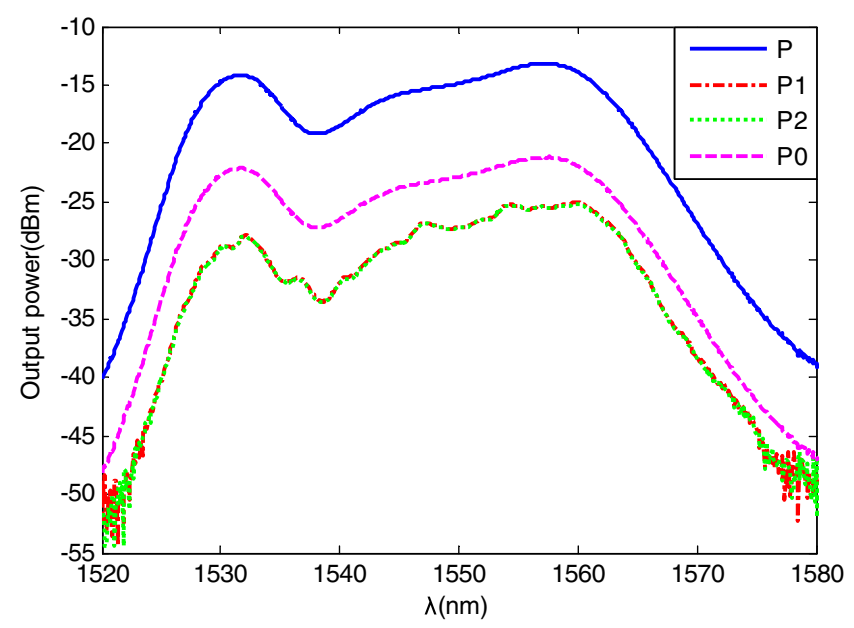

Fig. 15 Output power for the MMI coupler when $w=6 \mu \mathrm{m}$.

The output power of the MMI coupler when the center wavelength ranged from 1.52 to $1.58 \mu \mathrm{m}$ is demonstrated in Fig. 15, where the maximum excess loss is $0.8233 \mathrm{~dB}$. The experimental results show that the designed coupler has a wide range of wavelength responses.

\section{Analysis of the Designed Couplers}

The simulated and experimental results indicate that X coupler has a lower fabrication tolerance and a relatively larger size. Its splitting ratio is highly sensitive to the parameters of the coupling region. Given the same footprint, a CGC has relatively smaller loss and nonuniformity. Thus, CGCs can be used as alternatives to $X$ couplers. However, a CGC depends greatly on the wavelength and is sensitive to polarization. MMI couplers have several advantages such as their compact construction, low loss, simple fabrication technique, high fabrication tolerance, high uniformity, and small size. Thus, MMI couplers are widely used in planar lightwave circuits. As mentioned in Sec. 1, Chen's coupler ${ }^{2}$ has advantage of a wide bandwidth, but its footprint is large. The respective couplers designed by Yang ${ }^{3}$ and Tanaka ${ }^{4}$ have small footprints but have relatively large excess loss and nonuniformity. Our MMI coupler ( $w=6 \mu \mathrm{m}$ ) features the combined advantages of low excess loss and high uniformity, with a small footprint and wide bandwidth.

\section{Conclusion}

This paper introduces the fabrication and experimental results of a $2 \times 2$ coupler designed with silicon photonic nanowires for the AWG demodulation integration microsystem. Three kinds of $2 \times 2$ couplers are fabricated and tested. Compared with the CGC and the X coupler, the MMI coupler has the advantages of a smaller footprint, lower loss, wider bandwidth, and high fabrication tolerance. When the center wavelength is $1.55 \mu \mathrm{m}$, the designed MMI coupler, with a total footprint of $6 \times 100 \mu \mathrm{m}^{2}$, has an excess loss of $0.5423 \mathrm{~dB}$ and a uniformity of $0.0063 \mathrm{~dB}$ according to the experimental results. The maximum excess loss of this coupler is $0.8233 \mathrm{~dB}$ within the range of 1520 to $1580 \mathrm{~nm}$. Thus, the MMI coupler can meet optoelectronic integration requirements. 


\section{Acknowledgments}

Work supported by the National Natural Science Foundation of China (Grant Nos. 61177078, 60877049) and the Specialized Research Fund for the Doctoral Program of Higher Education of China (Grant Nos. 20101201120001).

\section{References}

1. B. Jalali and S. J. Fathpour, "Silicon photonics," Lightwave Technol. 24(12), 4600-4615 (2006).

2. W. Chen et al., "Broadband directional couplers fabricated in bulk glass with high repetition rate femtosecond laser pulses," Opt. Lett. 16(15), 11470-11480 (2008).

3. L. Yang et al., "Compact $2 \times 2$ tapered multimode interference couplers based on SU-8 polymer rectangular waveguides," Appl. Phys. Lett. 93(20), 203304 (2008).

4. D. Tanaka, Y. Ikuma, and H. Tsuda, "Comparative simulation of three types of 3-dB coupler using a Si wire waveguide," in 2010 Third International Conference on Communications and Electronics (ICCE), Nha Trang, Vol. 25, pp. 389-393 (2010).

5. R. Halir et al., "Silicon cross-connect filters using microring resonator coupled multimode-interference-based waveguide crossings," Opt. Express, 16(12), 8649-8657 (2008).

6. H. Li et al., "Design optimization and comparative analysis of siliconnanowire-based couplers," IEEE Photon. J. 4(5), 2017-2026 (2012).

7. $\mathrm{H}$. Li et al., "Highly compact $2 \times 2$ multimode interface coupler in silicon photonic nanowires for array waveguide gating demodulation integration microsystem," Opt. Laser Technol. 47, 366-371 (2013).

8. T. Le and L. W. Cahill, "The design of $4 \times 4$ multimode interference coupler based microring resonators on an SOI platform," $J$. Telecommun. Inform. Technol. 2, 58-62 (2009).

9. D. Dai and S. He, "A silicon-based hybrid plasmonic waveguide with a metal cap for a nano-scale light confinement," Opt. Express 17(19), 16646-16653 (2009).

10. K. Solehmainen et al., "Adiabatic and multimode interference couplers on silicon-on-insulator," IEEE Photon. Technol. Lett., 18(21), 2287-2289 (2006).

11. T. Amemiya et al., "Nonunity permeability in metamaterial-based GaInAsP/InP multimode interferometers," Opt. Lett. 36(12), 2327-2329 (2011).

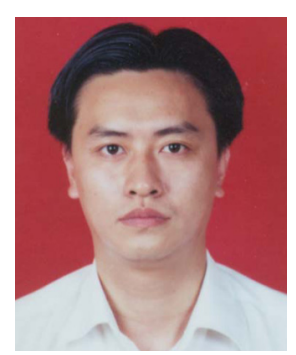

Hongqiang Li received his $\mathrm{BE}, \mathrm{MSc}$ and $\mathrm{PhD}$ degrees from Tianjin Polytechnic University, China. He is a vice professor in the School of Electronics and Information Engineering, Tianjin Polytechnic University. His research interests lie in the area of optical fiber grating sensor demodulation and optoelectronics integration.

Xiaye Dong is working on her Master's degree in the School of Electronics and Information Engineering, Tianjin Polytechnic University, China.

Yaoting Bai is working on his Master's degree in the School of Electronics and Information Engineering, Tianjin Polytechnic University, China.

Enbang $\mathbf{L i}$ is a professor in Centre for Ultrahigh bandwidth Devices for Optical Systems (CUDOS), University of Sydney, Australia.

Yang $\mathrm{Li}$ is working on his Master's degree in the School of Electronics and Information Engineering, Tianjin Polytechnic University, China.

Yu Liu is working on her Master's degree in the School of Electronics and Information Engineering, Tianjin Polytechnic University, China.

Wenqian Zhou is working on his Master's degree in the School of Electronics and Information Engineering, Tianjin Polytechnic University, China. 